\title{
RESEARCH OF SLUICE MONITORING SYSTEM BASED ON GPRS AND PLC
}

\author{
Qiulan $\mathrm{Wu}^{*}$, Yong Liang, Xia Geng, Wenjie Li, Yanling Li \\ School of Information Science and Engineering, Shandong Agricultural University, Taian, \\ China, 271018 \\ * Corresponding author, Address: School of Information Science and Engineering, Shandong \\ Agricultural University, Taian, 271018, Tel: +86-0538-8249755, Fax: +86-0538-8249755, \\ Email:wqlsdau@163.com
}

\begin{abstract}
The method of sluice control applied to reservoir is executed manually in field at present. To fix this problem, sluice remote monitoring system based on GPRS and PLC is provided. Some advanced technologies used in system are discussed. The system structure and principle are introduced. The system realizes wireless network connections between local control unit and center control unit based on GPRS technology, and it provides remote monitoring of reservoir sluice with a new technique.
\end{abstract}

Keywords: General Packet Radio Service, Programmable Logic Control, Sluice, Remote Monitoring

\section{INTRODUCTION}

At present, the field manual operation or field automatic operation is applied to sluice control in XueYe reservoir. Whenever it is of the violent storm or the hot sun, the operator must operate system on the dam, and record the system movement condition. The operator records the open time, position, stop time, stop position, etc of the sluice, and regularly process the data and summarize the information. The manual mode can't satisfy the requirements of development.

With the development of control theory, communication, computer technology and network, putting remote measurement and control, wireless communication and network technologies into sluice's control is the main 
stream of the reservoir sluice control system. The monitoring way of sluice is changed from individual centralized structure control to the hierarchical structure control (Wu et al., 2002).

This paper proposes a sluice remote monitoring system based on GPRS (General Packet Radio Service) and PLC (Programmable Logic Control). Its hardware and work principle are illustrated. The system implements automatic measurement and control of ascending height of the sluice, manual and automatic control of headstock gear, collection, data process and transmission of real-time information. The remote automatic control of sluice is an important part of water conservancy modernization. It provides a better tool to save water and energy, to manage water resources and optionally dispatch accomplished automatic measurement and control of ascending height of the brake (Dai, 2002; Zhang et al., 2002; Han et al., 2003).

\section{INTRODUCTION TO GPRS}

GPRS transmits data using grouping exchange with high efficiency. GPRS mobile telecommunication network is formed by adding nodes to the public communication telecommunication network, SGSN (Server for GPRS Support Node) and GGSN (Gate GPRS Support Node). It adopts grouping data exchange and provides users connection in the form of mobile grouping IP or x.25. It has same frequency slot, bandwidth, retransmission structure, wireless modulation, frequency-hopping and data frame of TDMA as GSM. GPRS can be divided into two parts: wireless access and the main network. The wireless access part is responsible for communication between mobile node and BBS (Base Station Subsystem) while the main network is responsible for communication between BBS and router of the standard digital communication network.

The advantages of GPRS are:

(1) No extra network needed, only application to be user of the public mobile communication network.

(2) The coverage of the public communication network is huge and the public communication network is extendible.

(3) Fee can be base on the usage or on the monthly plan.

User can be online all the time and fee is charged according to the data amount. When there is no data transmission, even the user is online, no fee is charged. Or, user can pay a monthly fee without usage limitation.

(4) Transmission speed is high.

Grouping data exchange is the necessary condition for data transmission and enhancement of the user capacity. 
Thus, wireless communication network making use of the public mobile communication network is low-cost, extendible, non-constraint, low-error and stable system with advancement and standards. GPRS is especially suitable for low data rate with high usage frequency communication like transformation of the data relevant to water quality (Wavecom Company, 2001).

\section{INTRODUCTION TO PLC}

PLC is a programmable controller. PLC storages instruction in the internals such as logical operator instructions, sequential control instructions, counting instructions and arithmetic operations instructions. PLC can control various types of mechanical equipment or production process through Digital or analog input and output. PLC has the function of network communication. It can exchange information between PLC and PLC, PLC and host computer, PLC and other intelligent devices. It can form an integrated, separately centralized control. Major PLC has the RS-232 interface and the interface which can support individual communication protocol.

PLC can adapt to the bad environment easier than single-chip processor and computer acting as filed controller. It has the advantages of convenient configuration, reliable remote communications and easy maintenance.

\section{SYSTEM DESIGN}

The layered distributed open architecture is adopted in the sluice Remote Monitoring System. The System consists of three parts: monitoring center, GPRS network and field controller (Fig. 1).

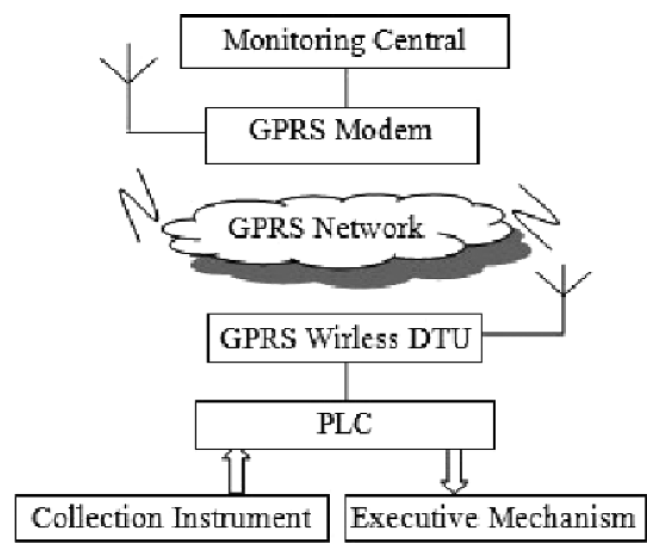

Fig. 1. Function architecture of system 


\subsection{Field Controller}

The main part of the field controller is PLC which collects the information and controls sluice. The data collection instrument of Field controller collects the real-time information such as ascending height of the sluice, water level, temperature and Humidity of sluice chamber. The information of sluice is coded by the coding program in PLC. PLC forwards it to GPRS wireless DTU (Data Terminals Units) through the RS232 communication interface. The data is processed and packaged by Embedded Processor of GPRS wireless DTU, which is transmitted to the data processing and monitoring center through wireless GPRS network.

The Control part is the core of field controller. The control system consists of control procedure of the sluice, man-machine interface of the scene operation, warning devices etc. The system mainly includes PLC, intermediate relays, man-machine interface board (switches, buttons, signs, show devices, alarm devices).

The command coming from sluice monitoring software of monitoring center is transmitted to GPRS wireless DTU through GPRS wireless modem and then retransmitted to PLC through RS232 communication interface. The code of command is translated by translation software of PLC. Then PLC completes corresponding control operation through calling automatically corresponding sluice control program.

Electric control mainly consists of electric equipments such as air switch, $\mathrm{AC}$ contactor, and intermediate relay. Automatic (manual) dial switch on the control cabinet can shut off or connect two different circuits. Electric control unit has the function of multiple protections. Motor overload protector can automatically shut off power in order to protect equipment when motor is overload. Limit switch can automatically shut off power of headstock gear in order to protect motor when height of sluice is out of limit location. It can avoid running abnormally of motor because of disorder phase sequence of three-phase power supply.

The system provides three control modes: manual control mode in field, automatic control mode in the scene, automatic remote control mode. Manual control mode in the scene has the highest priority. The remote operation is invalid and sluice only response to operation buttons of PLC cabinet when manual operation is selected. It guarantees that the dispatch of the water is normal when remote monitoring system is failed or in maintenance.

On the filed automatic control mode, setting the prospective height of the sluice on the Touch-screen, PLC can track and monitor the height of the sluice. The sluice stops when it reaches to the prospective height.

Selecting "PLC Automation" on the PLC control cabinet and "remote" on the remote monitoring computer, inputting the height of sluice with keyboard, click increase or decrease buttons with mouse, the sluice 
automatically runs, and it can stop when it reaches the prospective height. At the same time the operation button is invalid on the PLC control cabinet. The ascending or descending process of the sluice is showed on the image surveillance computer in the monitoring center (Liu et al., 2004; Zhang et al., 2007; Shi et al., 2007; Zhang et al., 2003; Huang et al., 2003).

\subsection{Monitoring Center}

Monitoring Center consists of server, monitoring computer, manage computer and image surveillance computer.

The server of monitoring center applies for assigning fixed IP address, using of DDN special line provided by mobile communication company to connect with GPRS network. Because DDN special line could provide relative high bandwidth, when the number of data collecting sites increased, monitoring center could meet the need without enlarging the capacity.

After receiving the data transferred from GPRS network, the server will firstly verify, and then transfer the data to the main control computer. The system will restore and process the data.

The function of monitoring center as follows:

(1) Remote Control

Monitoring center can transfer ascending or descending command of sluice.

(2) Surveillance Running

The image surveillance computer browses the website embedded in the video server. The picture of sluice is showed on the website. Camera and scanner can be adjusted remotely.

(3) Statistics and Print

Do statistics on the running of sluice and various performance indicators; print various tables of events and operation.

(4) Accident Analysis and Fault Diagnosis

After accident, accident analysis and anti-accident measures can be done according to a list of accidents, operating records and accident record. At the same time online real-time diagnosis can be done such as the front monitoring diagnosis, communication interface diagnosis, network interface diagnosis and computer equipment diagnosis.

\subsection{GPRS Network}

The data transmission uses GPRS wireless network. GPRS network communicates with DTU through GPRS modem, a product from Hongdian Company in a single-point to multi-point manner. There are several water data sampling instruments whose data are packed into IP packages and transmitted to the data processing and monitoring center through GPRS 
wireless network. GPRS network assigns fixed IP addresses within information center, according to the IP address assigned DTU set up data pathway with the information center.

GPRS DTU based on GPRS network of Chinese mobile has advantages of higher reliability and better anti-interference ability. It can connect computer, RTU, PLC, GPS receiver, digital cameras and data terminals through RS232 interface. It can support high-speed data transmission up to $171 \mathrm{Kbps}$. The function of remote diagnosis, testing and monitoring can meet the needs of data acquisition and control between control center and a large number of remote sites (Shenzhen Hongdian Technologies Co., Ltd. 2002).

\section{SOFTWARE DESIGN}

The software adopts modular design. It is easy to debug, modify, promotion and expand. The program module mainly includes automatic control module of sluice, the acquisition and process module of the sluice's height, the acquisition and process module of water level, the fault diagnosis and Analysis module etc.

\section{CONCLUSIONS}

After onsite simulation in irrigation district and hydropower plant of the reservoir, the system runs stably and dependably, and meets the requirement of remote monitoring. But it is still not satisfying for control of the floodreleasing sluice. The system needs further improvement.

The sluice remote monitoring system based on GPRS and PLC achieves automatic control of sluice. It can make the better use of water resource and improve the utilization rate of water energy. It is an important measurement to implement automatic and information China's water conservancy projects. It not only greatly enhances the automatic control capacity of sluice and reduces labor intensity and the number of duty, but also receives a lot of economical benefits.

\section{ACKNOWLEDGEMENTS}

This study has been funded by Shandong Water Conservancy Bureau. It is supported by technology special fund project of Shandong water resources (Number: 200357), and also supported by Shandong Agricultural University. Sincerely thanks are also due to XueYe reservoir for providing the data for this study. 


\section{REFERENCES}

Dai Shangan 2001, The Control of Floodgates in Water Conservancy Projects, Computer \& Digital Engineering, 29:1-6 (in Chinese).

Han Bing, Li Fenhua 2003, Application of GPRS technology in data acquisition and monitoring system, Electronic Technology, 19:26-29 (in Chinese).

Huang Xu, Zhao Ying 2003, Applications of PLC Programmable Controller and Supersonic Positioner for the Pressure-adjusting Well Gates Control of Hydroelectric Power Plant, Northeastern Electric Power Technology, 42-43 (in Chinese).

Liu Shubo, Xu Zhanguo 2004, Design and Realization of Sluice Automatic Control at the Head of Canal in Doushan Irrigation District, China Rural Water and Hydropower, 21-23 (in Chinese).

Shenzhen Hongdian Technologies Co., Ltd. User manual of H7000 GPRS Wirless DDN data terminal, 2002.

Shi Yitan, Gu Zhong bi 2007, Application of Simons S7-200 in the remote gate control system, Jilin Water Resources, 8-9 (in Chinese).

Wavecom company, GPRS User Guide, 2001

Wu Jianchun, Jiang Zhizhao 2002, Improvement of Water Intake Gate Control System of Generating Sets at Wuxijiang Hydropower Plant, Dam Observation and Geotechnical Tests, 26:55-60 (in Chinese).

Zhang Peng, Liu Qing, Zhang Zhixiu 2007, The reservoir gate monitoring and control system based on Fieldbus, Industrial Instrumentation \& Automation, 50-55.

Zhang Rentian, Xiao Jian, Ju Maosen 2002, Key Techniques of Real Time Automatic Control and Optimal Operation System of Irrigation Districts, China Rural Nater and Hydropower, 9-11 (in Chinese).

Zhang Zhixiu, Zhang Peng, Wang Zengyu 2003, A Kinds of the Reservoir Gate Monitoring and Control System, China Instrumentation, 33-35 (in Chinese). 